\title{
EDITORIAL
}

\section{Stress: a global multidimensional common denominator}

\author{
Uriel Halbreich (1)
}

\begin{abstract}
Uriel Halbreich, MD, is Professor of Psychiatry and Director of BioBehavioral Research at Jacobs School of Medicine and Biomedical Sciences, University of Buffalo, State University of New York (SUNY-AB), USA. He is the Founding Chair of the World Psychiatric Association Section on Interdisciplinary Collaboration. He was the Founding President of the International Association of Women's Mental Health and is a past President of the International Society of

Psychoneuroendocrinology. Correspondence Uriel Halbreich. Email: uhalbreich@gmail.com
\end{abstract}

First received 12 Jan 2021 Final revision 4 Feb 2021 Accepted 5 Feb 2021

\section{Copyright and usage}

(C) The Author, 2021. Published by Cambridge University Press on behalf of The Royal College of Psychiatrists

\section{SUMMARY}

The manifestations of stress are diverse and culturally and individually determined, but it is present in every culture, every socioeconomic level and - it is global. Introducing a special BJPsych Advances issue on stress, this editorial outlines research questions to be examined and first actions to be taken in the multidimensional sphere of stress, highlighting the need for a collaborative interdisciplinary approach in both research and practice.

\section{KEYWORDS}

Stress; globalisation; well-being; crisis; pandemic.

In the midst of the global crisis of the COVID-19 pandemic, we need to be reminded that crisis is the optimal opportunity for taking a well-rounded perspective. If followed by resourceful actions, the acute need to survive can open minds and strengthen wills to resolve that 'enough is enough' and 'this should not happen again'. The realisation that the same hardships are shared by many others may increase willingness for cooperation. Stress can give rise to conflicts, but as a common denominator it may enhance a spirit of unity to take the best road to overcome stressors and crises and to move closer to the ideal of well-being, safety, prosperity and happiness - together.

A historic modern departure point for a promising resolve for unity may be seen in the Second World War. At the depth of threat, the Allies decided to cooperate to solve the immediate crisis and prevent its recurrence. Following the war, the United Nations (UN) was created and one of its first actions was the establishment of the World Health Organization (WHO). All participating nations arrived at a global consensus that 'health is a state of complete physical, mental and social wellbeing'. The WHO also defined mental health as 'a state of well-being in which every individual realizes his or her own potential, can cope with the normal stresses of life, can work productively and fruitfully, and is able to make a contribution to her or his community' (World Health Organization 2005). In the reality of the 21st century, the 'normal stresses of life' include a series of frequent disasters and natural and man-made acute crises, on top of chronic grinding day-to-day economic struggles, interpersonal and social frictions and accelerated change in almost all aspects of life. This raises the question: 'Is stress normal?'. If it is, then how may we move from a negative distress to a more positive 'eustress' (Selye 1976).

\section{Questions in need of answers}

The pandemic-induced stress illuminates the globalised vulnerability to stressors as well as the impact on all levels of society: international, national, local communities, interest groups, families and individuals. It also demonstrates the diversity of responses, from unifying to divisive, from victimisation to altruistic heroism, from resilience to collapse of all systems, from hope to despair. The differences raise questions that are in need of operationalised solutions:

- Why do some societies respond to threats with unity and productive actions while others disintegrate?

- What is the role of leadership, how should it be executed and when?

- What is the optimal stress-reducing balance between individuals' self-interests and benefits to society?

- How can we prevent or reduce the next crisis? What are the financial, social and emotional costs of that prevention?

- Is there a community post-traumatic stress disorder (C-PTSD)? Can it be prevented?

- Why do some individuals, and societies, perceive an event as traumatic and respond with PTSD while others are resilient and even strengthened?

- Can stress be eliminated? Can community resilience be enhanced? How?

On a clinical public health level:

- Can vulnerable populations be identified prior to a crisis? This is important especially in places where disasters are so frequent that they are a predictable part of life, such as regions prone to floods, hurricanes, and violent tribal or border conflicts.

- Can vulnerable individuals - those with low resilience - be identified within the vulnerable population? 
- Can a cost-effective easily accessible mass-produced biological test for low resilience and stress be developed?

- Are there gender differences in stress responses? Why?

- Are there ethnic differences in resilience? Can they be distinguished from socioeconomic factors? Should they be?

The list of stress-related questions is almost endless. Indeed, as in any productive investigation, elaboration of one question opens the door to new ones. What is apparent is that operational knowledge of stress should be multidimensional and interdisciplinary. It should be integrative and comprehensive, acknowledging the complexity and applying innovative approaches to make it seem 'simple' and achievable.

Essentially, we should move from multiple separate 'focused' fields to a well-rounded multidimensional sphere of stress.

\section{Tackling the multidimensional sphere of stress}

As an essential step to understanding and combating stress and stress-related disorders there is a need for interdisciplinary collaborative partnerships (Halbreich 2019). It is not only that stress is globalthe sphere of stress is multidimensional and should be conceptually and practically well-rounded. A unidimensional field in which point A influences adjacent point B would not provide for a relevant operational answer because each change in the systems induces many other changes, and not all of them can be predicted or controlled.

As has been demonstrated by the COVID-19 pandemic, a change in one health parameter may cause profound changes in the economy, infrastructure, social services systems, interracial relationships, international relations, technological priorities and politics (among many other areas). Therefore, interdisciplinary partnerships are needed at a conceptual level as well as a very practical problem-solving level. This creates a management problem. Such interdisciplinary groups are routinely managed in for-profit corporations, where a product requires input from professionals with diverse expertise. Interdisciplinary groups should also be established in the public-benefit sector. Not-for-profit interdisciplinary task forces could learn from the input of business executives, who might even be integral partners of the group. An academic-industrygovernment cooperation might be an intriguing structure that could create sufficient incentives to overcome political and control obstacles. At first the interdisciplinary group might function as an advisory board, discussing the conceptual issues and their ramifications for planning. The planning would involve increasing levels of management and political considerations, leading to operational teams.

An initial minimally controversial issue might be screening for vulnerable populations. This epidemiological exercise would assess parameters such as gender, age, income, education, housing, occupation, employment, health indicators and other socioeconomic parameters. Epidemiological population-based screenings are very important in developing and emerging economies but the consequences of the COVID pandemic suggest their importance in established economies as well.

It is hypothesised that vulnerable populations would include pregnant women, very-low-income and poverty-stricken neighbourhoods, high-density urban housing and locations experiencing repeated recent disasters. Minorities and immigrants may also be more vulnerable to the consequences of stress. However, initial epidemiological studies of stress should be broad enough to uncover unexpected segments of the general population.

An important question with a needed and possible solution is: Once vulnerable populations, situations and locations are identified, who are the individuals with low resilience, whose resilience should be enhanced, and who are the people with high resilience who may be trained to be first responders?

A straightforward subsequent question with a complicated set of answers is: What makes an individual vulnerable to negative consequences of stressful events? Geneticists are making a good progress on that question, continuously demonstrating genes that are involved in mental symptoms in general or in specific symptoms (Caspi 2013). However, epigenetics plays an important role, and this change may be transferred across generations (Yehuda 2018). An intriguing question that is still to be answered is whether epigenetics is cumulative through many generations. If so, is there a genetics of social history? Is a long history of oppression and discrimination or slavery inducing scars that last for generations? What is, or are, the interfaces between genetics, epigenetics, environment, economy and perceptions?

Furthermore, inflammation has been shown to be important in the processes of stress responses. How is it transferable? Being demonstrated across several stress-related disorders, is the hereditary chain specific to a certain organ or body system? Or might it be expressed as a particular stress-related disorder in one generation, but have a different expression in another? A similar question is pertinent to autoimmune disorders. Here the contribution of the environment is of importance because even within the same individual, symptomatic expressions may vary in different locations and at various life stages. 
The contribution to and involvement of multiple hormonal systems in stress and stress responses is beyond doubt. However, several unsolved questions should be confronted. First, what is the contribution of gonadal hormones to sex differences (if there are any) in stress responses? Are any such differences related to the interactions between brain stress mechanisms and gonadal hormones, especially oestrogen? These interactions are well-documented in animals and humans (e.g. Bangaser 2019; Goldstein 2019) but are they also gender-related, incorporating the sociocultural aspects of being a man or a woman and what is expected of each one?

Another presumption is that some hormones may be biomarkers for stress - in particular, elevated levels of cortisol have been considered. However, in my opinion no specific hormonal marker of distress has been confirmed so far. This is an important issue in the development of biological and physiological tests and diagnostic aids for elucidation of stress and stress-related disorders in humans.

\section{'All is predetermined, but the choice is given'}

In the multicultural context of stress, spirituality should be added to the equation. As early as Biblical times a sage declared that 'all is predetermined, but the choice is given'. Fate is not definitively determined by genetics, epigenetics, inflammation, economy, birth and beliefs. Vulnerability is just that - vulnerability. Each individual (and society) has a choice how to handle his/her/our vulnerability and take appropriate actions.

What are the 'appropriate actions'? First, a change of attitudes is needed. Conventional thinking and approaches would not lead us far enough. The articles in the current issue of BJPsych Advances demonstrate that we know many facts about stress and its consequences. Clinicians can apply them in day-to-day practice. However, we should realise that question marks outweigh exclamation marks. To get deeper into the sphere of stress we should try to know what we do not yet know and fill some of the gaps with open minds and innovative approaches. This is especially important for prevention of the consequences of stressors. To construct efficacious preventions of an unknown event or a predictable event whose timing may be surprising, flexibility of mind and adaptable actions are a must. Change is inevitable, and we should not be overwhelmed by it.

\section{Funding}

This research received no specific grant from any funding agency, commercial or not-for-profit sectors.

\section{Declaration of interest}

None.

\section{References}

Bangaser DA, Eck SR, Sanches EU (2019) Stress differences in stress reactivity, in arousal and attention systems. Neuoropsychopharmacology, 44: $129-39$.

Caspi A, Houts R, Belsky D, et al (2013) The p factor: one general psychopathology factor in the structure of psychiatric disorders? Clinical Psychological Science, 2: 119-37.

Goldstein JM, Tuben H, Foster SL, et al (2019) Sex differences in major depression and comorbidity of cardiometabolic disorders: impact of prenatal stress and immune exposures. Neuropsychopharmacology, 44 : 59-70.

Halbreich U, Schulze T, Botbol M, et al (2019) Partnerships for interdisciplinary collaborative global well-being. Asia-Pacific Psychiatry, 11(2): e12366.

Selye H (1976) Stress in Health and Disease. Butterworth-Heinemann.

World Health Organization (2005) Basic Documents, 45th edn. WHO, (https://apps.who.int/iris/handle/10665/43134).

Yehuda R, Lehrner A (2018) Intergenerational transmission of trauma effects: putative role of epigenetic mechanisms. World Psychiatry, 17(3): 243-57. 